between increased spiritual well being and a decrease in physical symptoms and psychological distress in terminal care.

An audit was undertaken to evaluate whether patient's spirituality was being assessed on admission to the inpatient unit at an adult hospice. The standard set for this audit was $80 \%$ and applied to both genders.

The method employed was a retrospective cohort audit of 30 patients, using their admission pro formas. The quantitative data was collected assessed by whether the spirituality section in the admission pro forma had been filled in, and gender.

From the data collected the results showed an average of $73.30 \%$ $(n=22)$ of patients having a documented conversation about their spirituality and needs on the admission form. Further analysis highlighted that, when compared with the average, the percentage of female admissions that considered their spirituality rose to $89.47 \%$ $(n=17)$. However for the men the results fell to $45.45 \%(n=5)$. The standard set in this audit was, on average, not achieved by the hospice. It is recognised that due to the limitation of a single cohort it restricted the opportunity for equal gender population samples.

The lower results for the male gender could be attributed to gender specific barriers to discussing spirituality and may be a training issue for the team members. Therefore the main recommendations of this audit were for further education for all members of the healthcare team. NICE guidelines advocate the use of the 'Marie Curie's competency levels for Spiritual care' as an educational framework. In addition a spiritual assessment tool, Faith Active Coping Treatment plan (FACT), has been identified that may be very useful in directing these conversations.

\title{
AN AUDIT TO EVALUATE THE ASSESSMENT OF SPIRITUALITY FOR PATIENTS ADMITTED TO WILLOW WOOD HOSPICE INPATIENT UNIT
}

Bethanie Ragsdell, ${ }^{1}$ Michael Tapley'. ' Manchester Medical School, Manchester, United Kingdom; ${ }^{2}$ Willow Wood Hospice, Ashton-under-Lyne

10.1136/bmjspcare-2014-000654.185

Palliative medicine entails a holistic approach to patient care. Spirituality is an important component and should be explored with terminally ill patients. Studies have demonstrated a link 